Barbara Stiebels (Leipzig)

\title{
Polysemie und Umdeutung satzeinbettender Prädikate
}

\begin{abstract}
Die Polysemie satzeinbettender Prädikate spielt eine wichtige Rolle für deren Einbettungsverhalten. Konkret wird gezeigt, dass Polysemie mit struktureller Ambiguität als Kontroll- vs. Anhebungsverb assoziiert sein kann (Beispiel drohen/versprechen) und dass NEG-Raising auf bestimmte Lesarten eines polysemen Verbs beschränkt sein kann. Des Weiteren wird beleuchtet, welche Faktoren die syntaktische Flexibilität satzeinbettender Prädiakte, d.h. das Einbettungspotenzial bzgl. der wichtigsten Satzkomplementtypen des Deutschen, begünstigen und welche Rolle dabei Umdeutungen (z.B. von bedauern zu ,mit Bedauern äußern'), die Polysemie induzieren, spielen. Alle betrachteten Phänomene deuten darauf hin, dass sie S-Selektion (semantische Selektion) eine zentrale Rolle in der Satzeinbettung spielt.
\end{abstract}

\section{Einleitung}

Während die Polysemie im nominalen Bereich bereits intensiv erforscht worden ist (siehe u.a. Pustejovsky/Boguraev (Hg.) 1996; Nerlich et al. 2003), ist der Polysemie von Verben und insbesondere der von satzeinbettenden Verben weitaus weniger Aufmerksamkeit geschenkt worden (siehe Viberg 1983 zu Perzeptionsverben).

Die Polysemie bei satzeinbettenden Prädikaten spielt eine wichtige Rolle für deren Einbettungsverhalten, was ich in diesem Papier exemplarisch beleuchten möchte. Zur Veranschaulichung möge das Verb absehen dienen, das die Lesarten ,ahnen' (1a), ,ignorieren' (1b), ,darauf verzichten' (1c/d) und ,beabsichtigen“ (1e) hat.

(1) a. Es ist noch nicht abzusehen, wann ein Urteil gesprochen werden könnte. (DWDS Zeit 2013) ${ }^{1}$

b. „Sieht man einmal davon ab, dass die Bayern traditioneller leben als die Berliner ...“ (DWDS TS 2002)

1 Korpusbelege sind dem DWDS-Korpus (Kürzel DWDS: www.dwds.de), dem DeReKo (Kürzel IDS), oder der ZAS-Datenbank (Kürzel ZDB; Stiebels et al. 2017) entnommen. 
c. Wir sehen derzeit davon ab, Polizisten dazu aufzufordern, die Anweisung zu ignorieren. (DWDS TS 2004)

d. Von einer erneuten Überprüfung der Schulleiter sieht Potsdam ab. (DWDS TS 2004)

e. Überhaupt hat es die Polizei darauf abgesehen, den Rasern das Fahrvergnügen zu verleiden. (DWDS BZ 1997)

Entscheidend für die Interpretation sind dabei die Argumentrealisierung und im Fall von ,ahnen' zusätzlich ein modaler Operator (entweder als Konstruktion $a b$ $z u$-sehen sein oder mit Modal wie in absehen können). Die Lesarten ,ignorieren“ und ,verzichten' treten jeweils mit von-Korrelat/PP auf, die Lesart ,beabsichtigen“ mit der im Deutschen seltenen Argumentrealisierung mit auf-Korrelat/PP und expletivem Objekt; diese Lesart liegt auch der Nominalisierung Absicht zugrunde. Die folgende Tabelle zeigt, dass sich die verschiedenen Lesarten jeweils in ihrem Einbettungsverhalten unterscheiden: Alle vier Lesarten sind mit einem nominalisierten Satzkomplement (NOML) kompatibel, keine mit Verbzweit-Komplement (V2). Während ,ahnen' und ,ignorieren' interrogative Komplemente (INTER) zulassen, treten die Lesarten ,beabsichtigen' und ,verzichten' auch mit Infinitivkomplementen (INF) auf.

Tab. 1: Lesartenspezifisches Einbettungsverhalten von absehen

\begin{tabular}{lllllll}
\hline Lesart & & dass & V2 & INTER & INF & NOML \\
\hline ,ahnen‘ & absehen können & $\checkmark$ & - & $\checkmark$ & - & $\checkmark$ \\
,beabsichtigen‘ & es darauf absehen & $\checkmark$ & - & - & $\checkmark$ & $\checkmark$ \\
,ignorieren‘ & davon absehen & $\checkmark$ & - & $\checkmark$ & - & $? / \checkmark^{2}$ \\
,verzichten‘ & davon absehen & $? ?$ & - & - & $\checkmark$ & $\checkmark$ \\
\hline
\end{tabular}

Dieses lesartenspezifische Einbettungsverhalten ist nicht auf absehen beschränkt, sondern bei vielen anderen satzeinbettenden Prädikaten ebenfalls zu beobachten. Nicht immer sind die Lesarten durch Argumentstruktur und/oder Argumentrealisierung unterschieden. So hat das Verb übersehen bei gleicher Argumentreali-

2 In der Interpretation, ignorieren' treten nominalisierte Satzkomplemente nur mit Verwendung von absehen als Partizip II auf (z.B. Machtpolitisch spielt die FDP, von der Wahl des Staatsoberhauptes abgesehen, im Bund keine Rolle. (DWDS BZ 2004)). 
sierung zwei Lesarten: die frequentere ,nicht bemerken“ wie in (2a) und die seltenere ,den Überblick haben' wie in (2b).

(2) a. Alle übersahen, welche Leistung er vollbracht hatte - da hat er sie schließlich selbst erwähnt. (DWDS BZ 2005)

b. Wirklich sprechend wäre dieses Material freilich erst, wenn man ganz übersehen könnte, wie sich diese Regelungen auf das wirkliche Wahlund Lernverhalten der Schüler auswirken. (DWDS Zeit 1987)

Die zweite Lesart tritt fast ausschließlich mit Interrogativkomplementen auf:

Tab. 2: Lesartenspezifische Einbettung bei übersehen

\begin{tabular}{llllll}
\hline Lesart & dass & V2 & INTER & INF & NOML \\
\hline ,nicht bemerken‘ & $\checkmark$ & $\checkmark$ & $\checkmark$ & $\checkmark$ & $\checkmark$ \\
,den Überblick haben` & - & - & $\checkmark$ & - & ?? \\
\hline
\end{tabular}

Diese beiden Polysemiemuster sind prädikatsspezifisch und entsprechen keinem allgemeineren Muster. ${ }^{3}$ Systematischer sind die verschiedenen Lesarten von Experiencer-Prädikaten, die sich aus den Alternationen bzgl. der Realisierung von Stimulus- und Experiencer-Argument ergeben. So hat das Verb ängstigen (wie eine Reihe von anderen Experiencer-Prädikaten) eine Variante, in der der Experiencer als Objekt und der Stimulus als Subjekt realisiert wird wie in (3a/b), und eine reflexive Variante mit Experiencer als Subjekt wie in (3c).

(3) a. Darüber hinaus ängstigt die Mittelständler, was die politische Linke unter einer Reform der Erbschaftssteuer versteht. (ZDB 22287: DWDS Zeit 1970)

b. ... mit welchem Recht unser Auswärtiges Amt mit schöner Regelmäßigkeit die bei uns lebenden irakischen Christen damit ängstigt, ihr Fluchtgrund sei mit dem Tod Saddam Husseins weggefallen (ZDB 25069: IDS nun 2007)

3 Es gibt neben absehen kein weiteres Verb mit den gleichen Argumentrealisierungsmustern; von den wenigen Verben, die sowohl Muster mit auf-PP/Korrelat als auch Muster mit von-PP/ Korrelat aufweisen, hat keines parallele Interpretationseffekte. Die Lesartenunterschiede bei übersehen finden sich selbst bei ähnlichen Verben (wie überhören) nicht. 
c. Niemand sollte sich in Deutschland ängstigen müssen, dass es Mord und Totschlag gibt, wenn der Euro eingeführt wird. (ZDB 10572: DWDS BZ 2000)

Auch hier sind Unterschiede im Einbettungsverhalten zu beobachten, wobei das Fehlen von V2-Sätzen in der ersten Variante nicht überraschend ist, da V2-Sätze nur sehr bedingt in Subjektposition auftreten:

Tab. 3: Lesartenspezifisches Einbettungsverhalten von ängstigen (V2-Komplemente jeweils im Konjunktiv)

\begin{tabular}{llllll}
\hline Variante & dass & V2 & INTER & INF & NOML \\
\hline Sachverhalt P ängstigt Experiencer & $\checkmark$ & & $\checkmark$ & $\checkmark$ & $\checkmark$ \\
Agens/Thema ängstigt Exp. mit P & $\checkmark$ & $\checkmark$ & & $\checkmark$ & $\checkmark$ \\
Exp. ängstigt sich (vor/um) P & $\checkmark$ & $\checkmark$ & $\checkmark$ & $\checkmark$ & $\checkmark$ \\
\hline
\end{tabular}

Eine - wie ich später zeigen werde - systematischere Form der Polysemie liegt bei Umdeutungen wie in (4) vor, bei der das faktive Verb bedauern als ,mit Bedauern äußern' uminterpretiert wird und somit die Selektion eines V2-Satzes ermöglicht:

Die Kommilitonin [...] bedauert, zwar seien „radikaldemokratische Forderungen nicht verwirklicht”, immerhin aber „Demokratie im großen und ganzen“. (ZDB 1446: DWDS K-Ze 1982)

Angesichts der Tatsache, dass sich im Deutschen trotz seines sehr reichhaltigen Inventars an satzeinbettenden Prädikaten bereits vielfältige Formen der Polysemie nachweisen lassen, ist es nicht unerwartet, dass in Sprachen, die nur über ein sehr kleines Inventar an satzeinbettenden Prädikaten verfügen, viele satzeinbettende Prädikate polysem oder vage sind. Bspw. gibt es in Navajo ein generisches Einstellungsprädikat (nízin), das in Abhängigkeit von Tempus und Modalpartikeln im eingebetteten Satz die Interpretation ,wollen', ,wünschen' oder ,denken' hat (Bogal-Allbritten 2016). Ein anderes sehr einschlägiges Beispiel findet sich mit dem Verb ver- ,sagen, denken, erzählen, befehlen, wollen‘ aus Neverver/Lingarak (Vanuatu); dessen konkrete Interpretation hängt von der Markierung mit dem epistemischen Suffix -bor (siehe (5a)), der Applikativmarkierung -ikh am Verb (siehe (5c/d)), der Präsenz von (Quasi-)Komplementierern (te bzw. i-ver, siehe $(5 b-d))$ und der Realis/Irrealis-Distinktion im eingebetteten Satz ab. 
(5) Neverver/Lingarak (Barbour 2012) ${ }^{4}$
a. Ni-ver-bor [ei i-vlem].
1SG.REAL-say-maybe 3SG 3SG.REAL-come
,I think/assert that he came'
b. Ei i-ver [te i-khitrokh mang
3SG 3SG.REAL-say COMP 3SG.REAL-see man.ANA
adr ati-vkhal].
PL 3PL.REAL-fight
,He $\mathrm{H}_{\mathrm{i}}$ said that he ${ }_{\mathrm{i}}$ saw the men fight.
c. I-ver-ikh na [i-ver
3SG.REAL-Say-APPL 1SG 3SG.REAL-Say
[nimokhmokh ang i-vu ij]].
female ANA 3sG.REAL-go ANT
,He told me that the woman has gone.'
d. $\mathrm{Ei}_{\mathrm{i}}$ i-ver-ikh ei $\mathrm{i}_{\mathrm{j}}$ [i-ver
3SG 3SG.REAL-Say-APP 3SG 3SG.REAL-Say
$\left[\mathrm{ei}_{\mathrm{j}} \quad \mathrm{im}-\mathrm{bbu} \sim \mathrm{vu} \mathrm{si}\right]$ ].
3SG 3SG.IRR-RED go NEG
,He told him not to go“
e. Mang i-ver me [im-delmus ar].
man.ANA 3SG.REAL-want just 3SG.IRR-whip 3NSG
,The man just wanted/intended to whip them.

Die Lesarten ,erzählen` und ,befehlen` werden durch die Realis-Irrealis-Distinktion unterschieden (siehe $(5 \mathrm{c} / \mathrm{d})$ ); die Lesart ,wollen' tritt in Kombination mit Irrealis und komplementiererloser Struktur (siehe (5e)) auf.

Die wenigen Beispiele aus dem Deutschen und Neverver verdeutlichen bereits, dass die Lesart eines satzeinbettenden Prädikats mit einer spezifischen Argumentstruktur bzw. Argumentrealisierung, einem spezifischen Komplementtyp (inkl. Wahl des Komplementierers), dem Verbmodus des eingebetteten Prädikats oder mit modalen Operatoren über dem satzeinbettenden Prädikat assoziiert sein kann.

Im Folgenden werde ich die Rolle der Polysemie satzeinbettender Prädikate für drei Bereiche beleuchten: Polysemie und die strukturelle Ambiguität als Kontroll- oder Anhebungsverb (Abschn. 2), die Rolle der Polysemie bei NEG-Raising (Abschn. 3) und der syntaktischen Flexibilität von satzeinbettenden Prädikaten

4 Abkürzungen: ANA - anaphor. Demonstrativum; ANT - Anteriorität ; APP - Applikativ, coMP - Komplementierer; IRR - Irrealis; NEG - Negation; NSG- Nicht-Singuar; REAL - Realis; RED Reduplikation; SG - Singular. 
(Abschn. 5). In Abschnitt 4 gehe ich zuvor kurz auf die Umdeutung (Coercion) von satzeinbettenden Prädikaten ein, die in vielen Fällen bei der V2-Selektion relevant ist.

\section{Polysemie und Kontrolle vs. Anhebung}

Die Polysemie von satzeinbettenden Prädikaten führt in einigen wenigen Fällen zur strukturellen Ambiguität als Kontroll- und Anhebungsverb. Einschlägiges Beispiel ist die vieldiskutierte Herausbildung einer Nicht-Sprechakt-Interpretation bei drohen und versprechen (siehe u.a. Askedal 1997; Reis 1997; Heine/Miyashita 2008). Diese Polysemie ist nicht nur aufgrund der syntaktischen Relevanz bei Infinitivkomplementen interessant, sondern auch, weil sie systematisch für eine Reihe anderer Sprachen nachgewiesen werden konnte: z.B. Englisch promise/ threaten (Traugott 1993); Spanisch prometer/amenazar (Cornillie 2004); Französisch promettre/menacer; Niederländisch beloven/dreigen (Verhagen 2000) und weitere Sprachen (siehe Heine/Miyashita 2008).

Die Beispiele in (6) illustrieren die Verwendung der beiden Verben als Kontrollverben mit Infinitivkomplement (jeweils Subjektkontrolle) und in Sprechaktinterpretation.

(6) a. $\mathrm{Er}_{\mathrm{i}}$ hatte dem Konzern ${ }_{\mathrm{j}}$ gedroht, $\left[_{-\mathrm{i}}\right.$ Lebensmittel zu vergiften und die Gefahr für die Kunden öffentlich zu machen]. (DWDS Zeit 2015)

b. Die First Lady $_{\mathrm{i}}$ verspricht den Wählern ${ }_{\mathrm{j}}, \mathrm{L}_{\mathrm{i}}$ alles zu tun, um einen Sieg von Donald Trump zu verhindern]. (DWDS Zeit 2016)

Ob ein Sprechakt als Drohung oder Versprechen interpretiert wird, hängt u.a. von der Bewertung der durch das Satzkomplement denotierten Situation für den Sprechaktadressaten ab. Bei positiver Bewertung wird der Sprechakt als Versprechen, bei negativer als Drohung charakterisiert. Bei beiden Verben ist nun überdies eine Verwendung möglich, in der die Bedeutungskomponente des Sprechaktes eliminiert ist, aber die prospektive Lesart eines positiven oder negativen Ausgangs der vom Satzkomplement bezeichneten Situation bewahrt bleibt. ${ }^{5}$ Demzufolge wird der Einsturz eines Gebäudes je nach Perspektive wie in (7a) (z.B. aus

5 In der Literatur gibt es eine umfangreiche Diskussion zur Interpretation der hier als „prospektiv“ charakterisierten Variante (epistemische/evidentielle/aspektuelle Lesart, siehe Zusammenfassung in Heine/Miyashita 2008). 
Perspektive des Hausbesitzers) oder (7b) (aus Perspektive von Personen/Institutionen, die das Gelände anderweitig nutzen wollen) dargestellt.
a. Das Gebäude droht einzustürzen. [Resultat negativ bewertet]
b. Das Gebäude verspricht einzustürzen.
[Resultat positiv bewertet]

In beiden Fällen liegt jeweils Anhebung vor, wie sich durch entsprechende Tests (u.a. Kombination mit unpersönlichen Verben wie regnen, Nichtpassivierbarkeit des Matrixverbs in diesem Kontext) nachweisen lässt. Traugott (1993) und Heine/ Miyashita (2008) zeichnen den semantischen Wandel, der zur Herausbildung der Anhebungsvariante führt, für Englisch bzw. Deutsch und andere Sprachen nach. Heine/Miyashita gehen davon aus, dass sich die Polysemie der beiden Verben über Sprachkontakt areal ausgebreitet hat, wobei nicht alle Sprachen die desemantisierte prospektive Lesart auch bei belebten Subjektreferenten zulassen (z.B. das Kind droht vom Stuhl zu fallen).

Teilweise ist auch zu beobachten, dass die Polysemie durch eine separate Lexikalisierung der Lesarten blockiert ist. Während bspw. das ukrainische Verb obicjaty ,versprechen' sowohl Kontroll- als auch Anhebungsverb sein kann, werden bei ,drohen' die Sprechaktlesart (po-grožuvaty) und die prospektive Lesart (za-grožuvaty) lexikalisch differenziert (Yuriy Kushnir, pers.).

Neben drohen/versprechen sind auch Phasenverben wie ,anfangen' und ,aufhören' in einer Reihe von Sprachen polysem und gleichfalls strukturell ambig. Ebenso lässt sich beobachten, dass ,wollen' in einigen Sprachen - in analoger Weise desemantisiert - mit unpersönlichen Verben kombiniert werden kann, womit eine funktionale Verwendung neben der als Kontrollverb etabliert wird, wie das spanische Beispiel in (8) und die Belege des Neuhochdeutschen in (9) illustrieren:

\section{Spanisch}

Parece que quiere llover.

scheinen.3SG comp wollen.3SG regnen.INF

,es scheint regnen $\mathrm{zu}$ wollen'

(9) a. Das ist doch Schade, daß es nicht mehr so recht schneyen und frieren will. (DWDS KK 1783)

b. Als es beispielsweise im Sommer 1990 in Frankreich nicht mehr regnen wollte, ... (DWDS Zeit 1992) 


\section{NEG-Raising}

Unter NEG-Raising (Horn 1978) versteht man die Eigenschaft bestimmter satzeinbettender Prädikate, bei Negation im Matrixsatz eine Interpretation der Negation als Satznegation des eingebetteten Satzes zuzulassen. In der Regel ergeben sich somit bei Negation des Matrixprädikats zwei Lesarten: die „hohe“ Lesart mit Negierung des Matrixsatzes/Matrixprädikats und die „tiefe“ Lesart mit Negierung des eingebetteten Satzes. (10b) gibt die NEG-Raising-Interpretation von (10a) wieder.

(10) a. Ich denke nicht, dass das Thema Drogen so wichtig ist. (DWDS BZ 2004)

b. $\Rightarrow$ Ich denke, dass das Thema Drogen nicht so wichtig ist.

NEG-Raising ist in der Regel auf eine kleine Klasse satzeinbettender Prädikate beschränkt. Zu den sprachvergleichend stabilsten NEG-Raising-Prädikaten gehören ,denken', ,glauben', und ,scheinen', wie Popp (2016) zeigen konnte. Sie unterscheidet in ihrer sprachvergleichenden Untersuchung von NEG-Raising-Prädikaten deshalb zwischen „starken“ und „schwachen“ NEG-Raising-Prädikaten. Erstere zeigen ein sprachübergreifend uniformes Verhalten als NEG-Raising-Prädikate, letztere (z.B. ,hoffen') dagegen ein sprachspezifisch abweichendes Verhalten.

Sprachübergreifend konsistent ist der Ausschluss von NEG-Raising bei faktiven Verben, wie das folgende Beispiel illustriert; (11b) stellt keine mögliche Interpretation von (11a) dar.

(11) a. Ich bereue nicht, dass ich an der Uni war, aber die letzten Semester hätten wirklich nicht sein müssen. (DWDS Zeit 2004)

b. $\nRightarrow$ Ich bereue, dass ich nicht an der Uni war.

Für die Polysemieproblematik besonders relevant ist nun das Verb erwarten, das eine allgemein prospektive (,denken, annehmen') und eine quasi-direktive Lesart (,verlangen') aufweist. Nur in der allgemein prospektiven Lesart erlaubt es NEGRaising (vgl. (12a/b) mit (12c/d)).

(12) a. Österreichs Finanzminister Hans Jörg Schelling erwartet nicht, dass aus Griechenland neue Reformvorschläge kommen. (DWDS Zeit 2015)

b. $\Rightarrow$ Der Finanzminister erwartet, dass aus Griechenland keine neuen Reformvorschläge kommen.

c. Die Menschen erwarten von der Politik nicht, dass sie alles hundertprozentig löst, (DWDS TS 2002) 
d. $\nRightarrow$ Die Menschen erwarten von der Politik, dass sie nicht alles hundertprozentig löst.

Auch in den von Popp untersuchten Sprachen erlaubt, erwarten' nur in der prospektiven Lesart NEG-Raising. Besonders interessant sind das spanische Verb esperar und das litauische Verb tikètis, die u.a. auch die Interpretationen ,hoffen“ aufweisen, aber nur in der Interpretation ,erwarten, denken“ NEG-Raising zeigen:

Tab. 4: NEG-Raising-Prädikate (Popp 2016/pers.); $\checkmark$ : NEG-Raising

\begin{tabular}{|c|c|c|c|c|}
\hline & ,wünschen' & ,hoffen' & $\begin{array}{l}\text {,erwarten, } \\
\text { denken" }\end{array}$ & $\begin{array}{l}\text {,erwarten, } \\
\text { verlangen' }\end{array}$ \\
\hline Spanisch & esperar & esperar & esperar $\checkmark$ & esperar \\
\hline Chinesisch & xīwàng $\checkmark$ & xīwàng $\checkmark$ & qīwàng $\checkmark$ & $?$ \\
\hline Italienisch & augurarsi & sperare & aspettarsi $\checkmark$ & aspettarsi \\
\hline Schwedisch & att önska & att hoppas & att förvänta $\checkmark$ & att förvänta \\
\hline Dänisch & at ønske & at håbe $\checkmark$ & at forvente $\checkmark$ & at forvente \\
\hline Norwegisch & å $ø$ ske & å håpe $\checkmark$ & å forvente $\checkmark$ & å forvente \\
\hline Litauisch & linkèti $\checkmark$ & tikètis & tikètis $\checkmark$ & tikètis \\
\hline Indonesisch & ingin $\checkmark$ & berharap $\checkmark$ & menduga $\checkmark$ & berharap \\
\hline Ungarisch & kíván $\checkmark$ & remél $\checkmark$ & $?$ & elvár \\
\hline Swahili & kutarajia & kutarajia & kutarajia $\checkmark$ & kutaka \\
\hline
\end{tabular}

Generell scheinen insbesondere Einstellungsprädikate ohne Präferenzkomponente NEG-Raising zu erlauben: In der prospektiven Lesart von erwarten hat der Einstellungsträger keine Präferenz für die vom Satzkomplement denotierte Situation, in der quasi-direktiven Lesart dagegen schon. Die sprachübergreifend konsistentesten NEG-Raising-Prädikate ,glauben', ,denken` und ,scheinen' drücken ebenfalls neutrale Einstellungen aus.

\section{Umdeutung (Coercion)}

Wie bereits in (4) illustriert können faktive Prädikate so umgedeutet werden, dass sie mit V2-Nebensätzen auftreten können. Eine analoge Umdeutung von bedauern zu ,mit Bedauern sagen' liegt in (13) bei der Verwendung von bedauern als Parentheseprädikat vor: 
Bis heute, bedauert Sick, hätten viele Frauen sich noch nicht von der Vorstellung gelöst, dass irgendwann ein Prinz käme, der alle Geldprobleme löst. (DWDS Zeit 2009)

Diese Umdeutung (englisch Coercion) induziert eine Polysemie des betreffenden Prädikats und spielt eine wichtige Rolle bei der Lizenzierung von V2Nebensätzen. ${ }^{6}$

Umdeutung ist insbesondere im Zusammenhang mit NP/DP-Objekten von satzeinbettenden Prädikaten wie enjoy/begin diskutiert worden (z.B. Pustejovsky 1995). Diese Prädikate selegieren ein ereignisdenotierendes Komplement wie in (14a). Da NPs (DPs) mit underivierten Substantiven als Kopf in der Regel nicht auf Ereignisse referieren, ist hier eine Umdeutung in eine ereignisdenotierende Entität erforderlich. Welches Ereignis jeweils inferiert werden kann, ergibt sich aus dem Kontext bzw. enzyklopädischem Wissen (Leseereignis in (14b), Fressereignis in (14c)). Modellierungen hierzu finden sich bspw. in Pustejovsky (1995) und Asher (2011).
a. Mary enjoyed/began [reading the book].
b. Mary enjoyed/began [the book].
c. The goat enjoyed [the book].

Der Umdeutungsmechanismus lässt sich gut bei Verben verdeutlichen, die nicht bereits inhärent satzeinbettend sind. Dazu möchte ich u.a. Lautemissionsverben bzw. mimikdenotierende Verben (z.B. grinsen) zählen. Lautemissionsverben unterscheiden sich dahingehend, ob sie das Hervorbringen eines Geräusches (z.B. rascheln, pochen, knirschen) oder die Existenz eines Geräusches (z.B. hallen, tönen) bezeichnen. Ein weiterer Parameter ist die Belebtheit der Lautemissionsquelle (z.B. Tierlautverben wie bellen vs. Verben wie bimmeln) und die Komplexität der Verbbedeutung, d.h. ob es neben der Geräuschkomponente noch weitere Bedeutungskomponenten gibt (z.B. einfache Verben wie piepen vs. komplexere Verben wie räuspern).

Die Umdeutung von Lautemissionsverben zu Bewegungsverben (siehe z.B. Levin/Rapoport 1988; Kaufmann 1995; Engelberg 2009) ist möglich, sofern die Lautemission kausal an die Bewegung gebunden ist oder beide auf die gleiche Ursache zurückgehen:

\footnotetext{
6 Eigentlich ist „Umdeutung“ eine etwas schwache Übersetzung von Coercion, da der erzwingende Charakter der Umdeutung nicht mit konnotiert ist.
} 
Neben der Umdeutung in ein Bewegungsverb kann ein Lautemissionsverb aber auch als Äußerungsverb umgedeutet werden, was dann entsprechende Satzkomplemente wie in (16) lizenziert. ${ }^{7}$

(16) a. ... quäkte der rachsüchtige Bush zurück, Chirac habe vor allem die Cheeseburger von gestern gemocht. (DWDS BZ 2004)

b. Geraldine Chaplin [...], die in jedes Mikrofon säuselte, wie sehr sie Berlin liebt. (DWDS BZ 2004)

c. Ich zischte, dass er nie mehr um diese Zeit bei mir klingeln soll. (DWDS BZ 2002)

Beide Formen der Umdeutung sind an die Eigenschaft des Deutschen gekoppelt, komplexe Verbbedeutungen generieren zu können, bei denen der Bewegungsmodus - oder genereller der Handlungsmodus - mit anderen Bedeutungskomponenten (hier Bewegung oder Vollzug einer Äußerung) verknüpft wird.

Für diese Verknüpfung von Bedeutungskomponenten sind in der Literatur u.a. Konzepte wie „Conflation“ (Talmy 1985) oder „Lexical subordination“ (Levin/ Rapoport 1988) vorgeschlagen worden. ${ }^{8}$ (17) skizziert eine mögliche Repräsentation des Umdeutungsprozesses: (17a) repräsentiert vereinfacht das Lautemissionsverb in seiner Ausgangsverwendung, wobei MANNER hier als Variable für die verbspezifische Art und Weise der Lautemission fungiert. Das Verb wird wie in (17) um ein Kommunikationsprädikat cOMMUNICATE erweitert, das gleichzeitig das Satzargument lizenziert.
a. $\lambda \mathrm{x} \lambda \mathrm{s}$ [EMIT_SOUND(x)(s) \& MANNER(s)]
b. $\lambda \mathrm{p} \lambda \mathrm{x} \lambda \mathrm{s}$ [EMIT_SOUnd(x)(s) \& MANneR(s) \& COMmunicATE(x,p)(s)]

7 Eine andere Form der Umdeutung von Lautemissionsverben bzw. mimikdenotierenden Verben ist die als faktiv-emotives Verb:

a. Er stöhnt darüber, dass ihm dieser angebliche Skandal mögliche Deals verhageln könnte. (DWDS TS 1999)

b. DDRler grinsen schadenfroh darüber, daß die bundesrepublikanischen Benzinpreise mittlerweile auch das östliche Niveau erreicht haben. (DWDS KK 1986)

8 Wie Talmy (1985) gezeigt hat, ist die Option, den Bewegungsmodus im Verb zu kodieren, einzelsprachlich parametrisiert. Germanische Sprachen (,satellite-framed“) erlauben dies, romanische (,verb-framed“) nicht. 
Troyke-Lekschas (2013) hat das Verhalten von 160 (157) Lautemissionsverben untersucht; für 60\% der Verben konnte sie Verwendungen als Sprechaktverben belegen. Für 30 Verben hat sie überdies Korpusuntersuchungen zum Einbettungsverhalten durchgeführt; dabei zeigte sich tendenziell folgende Implikationsbeziehung in der Ausweitung des Selektionspotenzials:

(18) Selektion bei Lautemissionsverben

direkte Rede $>$ V2-Nebensätze $>$ dass-Sätze $>$ (Interrogative $>$ ) Infinitive

Konkret bedeutet dies, dass Lautemissionsverben als Sprechaktverben umgedeutet zuerst bzw. frequenter in Konstruktionen mit direkter Rede auftreten. In ihrer Erweiterung des Satzselektionspotenzials treten sie dann mit V2-Sätzen, dann mit dass-Sätzen, teilweise mit Interrogativen und schließlich mit Infinitiven auf. Insbesondere Infinitive deuten auf eine entsprechende Usualisierung des umgedeuteten Lautemissionsverbs als satzeinbettendes Prädikat hin. ${ }^{9}$

Laut Wechsler (2017) treten Lautemissionsverben des Englischen nicht mit Interrogativkomplementen auf, die Fragesprechakte bezeichnen. Diese Beschränkung scheint tendenziell auch für das Deutsche zu gelten; in (19a) liegt eine Exklamativstruktur vor; in (19b) referiert das Interrogativkomplement auf einen Fragesprechakt, allerdings erscheint der Beleg sehr markiert.

(19) a. Hatte Thomas Gottschalk eingangs gekrächzt, welch grausiger Infekt ihn soeben niedergeworfen, aber nicht bezwungen habe ... (DWDS BZ 2003)

b. Dann hört ich die Haupttür am Hauptbau in ihren Angeln kreischen und ein Mann trat heraus in Schlafrock und Pantoffeln, ein dicklicher, und auf dem Kopf eine Schlafmütz, und krächzt, was ich wollt? (DWDS BZ 1998)

\footnotetext{
9 Eine Frage, die wiederholt gestellt wird, ist die nach der syntaktischen Integration der von Lautemissionsverben selegierten Sätze. Hier zeigt sich ein inkonsistentes Bild (und sicherlich gibt es Unterschiede zwischen den Verben).

i. Manchmal zischt er, dass sie an allem schuld sei. (DWDS Zeit 2008)

Wendet man einige der von Reis (1997) verwendeten Tests auf das Beispiel (i) an, so sprechen Bewegbarkeit in die Vorfeldposition, Variablenbindung und Verwendung als elliptische Antwort auf eine Frage für syntaktische Integration. Gegen syntaktische Integration sprechen die Unzulässigkeit von es-Korrelaten und die fragliche Bewegbarkeit in die Mittelfeldposition.
} 


\section{Syntaktische Flexibilität}

Wie bereits in der Einleitung exemplarisch deutlich gemacht wurde, ist das Einbettungsverhalten satzeinbettender Prädikate an spezifische Lesarten des Prädikats gebunden. In diesem Zusammenhang stellt sich auch die Frage, inwieweit die syntaktische Flexibilität eines satzeinbettenden Prädikats Ausdruck entsprechender Polysemie ist. Als syntaktisch flexibel gelten hier solche Prädikate, die mit mehreren oder sogar allen Satzkomplementtypen des Deutschen auftreten können; diskutieren möchte ich dies für dass-Sätze, Verbzweitsätze, Interrogativ-, Infinitiv- und nominalisierte Satzkomplemente.

Auf der Basis des in der ZAS-Datenbank dokumentierten Einbettungsverhaltens satzeinbettender Prädikate lässt sich festhalten, dass für ca. 25\% der 1.747 Prädikate der ZAS-Datenbank alle genannten Komplementtypen in Korpora nachgewiesen werden konnten. ${ }^{10}$ Generell zeigt sich folgende Distribution der Komplementtypen:

Tab. 5: Selektionsverhalten der satzeinbettenden Prädikate gemäß ZAS-Datenbank

\begin{tabular}{llllll}
\hline Komplementtyp & dass & V2 & INTER & INF & NOML \\
\hline Anteil lizenzierender Prädikate & $96 \%$ & $47 \%$ & $55 \%$ & $83 \%$ & $88 \%$ \\
\hline
\end{tabular}

Dass-Sätze können hierbei als Defaultkomplementationstyp gelten. Am restringiertesten sind V2-Sätze. Syntaktisch sehr flexibel ist bspw. das generische Kommunikationsverb mitteilen. Dieses Verb lässt unspezifiziert, wie der Kommunikationsweg erfolgt; beim V2-Satz in (20b) wird jedoch ein konkreter Sprechakt mit Redebericht zugrundegelegt. Anders als bei Verben, die zwingend auf einen konkreten Sprechakt referieren, kann das nominalisierte Satzkomplement auf den Kommunikationsinhalt referieren (siehe (20e)).

(20) a. Die Ärzte teilten ihm mit, dass er querschnittsgelähmt ist. (DWDS TS 2004)

b. Thierse teilte am Abend mit, er bedaure den Entschluss. (DWDS BZ 2003)

10 Bei der Berechnung der Prädikatszahlen in der ZAS-Datenbank habe ich die Ausdifferenzierung von Lesarten in der Datenbank durch separate Lexemeinträge ignoriert, da die Behandlung der Polysemie in der aktuellen Fassung eher tentativ und inkonsistent ist. 
c. Sotheby's teilte nicht mit, wem der Schmuck zuletzt gehört hatte. (DWDS BZ 2004)

d. Am Donnerstag teilte RTL mit, künftig ausführlich über Beachvolleyball zu berichten. (DWDS BZ 2005)

e. Man brachte uns in ein Büro, wo uns die Entlassung aus der DDRStaatsbürgerschaft mitgeteilt wurde. (ZDB 6593: DWDS K-Be 1994)

\subsection{Lizenzierung der verschiedenen Komplementtypen}

Wie bereits gesagt, repräsentieren dass-Komplemente den Defaultfall der finiten Satzkomplementation. Dies wird auch untermauert durch die Tatsache, dass es in der ZAS-Datenbank kein einziges Prädikat gibt, das mit allen anderen vier Komplementtypen auftreten kann, aber nicht mit dass-Sätzen (siehe Tab. 6). Vor diesem Hintergrund sind Implikationsbehauptungen (z.B. „alle V2-Einbetter bzw. alle Infinitiveinbetter selegieren auch dass-Sätze“) als wenig aussagekräftig zu betrachten, zumal sie empirisch nicht völlig korrekt sind, wie die Daten aus der ZAS-Datenbank belegen.

Dass-Sätze sind bei intensionalen Frageprädikaten nur sehr bedingt möglich (siehe unten). Ebenso erlauben einige ereigniskohärenten Prädikate (Stiebels 2010) keine dass-Komplemente (z.B. implikative Prädikate wie erdreisten, außerstande sein). Überdies werden dass-Sätze bei bestimmten Prädikaten vermieden, die direkte Rede und V2-Sätze präferieren (z.B. anflunkern, angeifern).

V2-Nebensätze werden typischerweise durch doxastische Einstellungs-, Gewissheits-, Äußerungs- und Präferenzprädikate lizenziert (Reis 1997); faktive Prädikate sowie viele inhärent negative Prädikate selegieren in der Regel keine V2-Sätze; allerdings kann eine Umdeutung wie in (4) das Einbettungsverhalten verändern.

Für die Lizenzierung von Interrogativkomplementen (siehe Karttunen 1977; Dipper 1997) kommen neben Frageprädikaten auch Wissensprädikate (z.B. wissen), Wissenserwerbsprädikate (z.B. erfahren), Äußerungsprädikate, Verben des Entscheidens, Vermutens und der Meinungsbekundung, Relevanzprädikate (z.B. wichtig sein) und Verben zur Bezeichnung von Dependenzrelationen (z.B. abhängen von) in Frage. Faktive Prädikate erlauben nur W-Komplemente, keine eingebetteten Polaritätsfragen.

Deutsch ist bzgl. Infinitivkomplementen weniger restriktiv als viele andere Sprachen, was sich bspw. daran festmachen lässt, dass auch faktive Verben mit Infinitivkomplement auftreten können. Allerdings wird dann häufig das Infinitivkomplement temporal wie in (21a) über das infinite Perfektauxiliar oder modal wie in (21b) über das Modalverb erweitert: 
(21) a. Wir fragten, ob er denn bereue, 1974 zurückgetreten zu sein. (ZDB 2223: DWDS BZ 1994)

b. Es ist das erste Mal in meinem Leben, dass ich bereue, nicht tanzen zu können. (DWDS Zeit 2013)

Strukturell sind Infinitivkomplemente bei intensionalen Frageprädikaten ausgeschlossen, da das Standarddeutsche keine eingebetteten W-Infinitive zulässt. Weitere strukturelle Bedingung ist die Etablierung einer Kontrollrelation, sofern nicht alternativ eine Einbettung durch ein Anhebungsverb vorliegt. ${ }^{11}$

Infinitivkomplemente können auch bei inhärenten oder umgedeuteten Äußerungsprädikaten auftreten, allerdings deutlich infrequenter. Auch hier ist in den meisten Fällen eine temporal/modale Erweiterung des Infinitivkomplements zu beobachten:

... bei 180 Volt schreit er, den Schmerz nicht länger ertragen zu können. (DWDS Zeit 1969)

Nominalisierte Satzkomplemente unterliegen der strukturellen Beschränkung, dass das satzeinbettende Prädikat dem Satzargument Kasus zuweisen können muss. Verben wie sich weigern, die dem Satzargument keinen Kasus zuweisen, erlauben dementsprechend auch kein nominalisiertes Satzkomplement. Korpusbelege wie der folgende dürften für sehr viele Sprecher inakzeptabel sein (da hier augenscheinlich eine Verwechslung mit sich wehren vorliegt und somit auch der implikative Charakter von sich weigern verlorengeht).

Bauern, bei denen nichts mehr zu holen war oder die sich lange gegen eine Herausgabe von Nahrungsvorräten geweigert hatten, wurde der »Schwedentrunk« verabreicht. (ZDB 17237: IDS nuz 2004)

11 Nicht immer kann eine direkte Kontrollrelation etabliert werden. Zulässig ist optionale oder obligatorische implizite Kontrolle (z.B. bei anordnen), mitunter erfolgt die Selektion eines Infinitivkomplements nur in Kombination mit einem Kontrollwechselkontext (Stiebels 2010; Brandt/ Trawiński/Wöllstein 2016) wie im folgenden Beispiel (Passivierung des eingebetteten Verbs):

a. Doch durch Zufall entdeckt sie, als Köder für ein politisches Komplott mißbraucht zu werden. (ZDB 10739: DWDS BZ 1994)

Bei einigen (Dis-)Präferenzprädikaten (wie dafür/dagegen sein) ist auch nicht-lokale Kontrolle möglich. 
Faktive Prädikate und Prädikate, die ereignisbezogene Komplemente selegieren (z.B. absagen), gehören zu den kanonischen NoML-Lizenzierern. Die meisten Äußerungsprädikate treten nicht mit nominalisierten Satzkomplementen auf, wobei man nicht klar trennen kann, ob dies als Kasusrestriktion zu interpretieren ist oder als fehlende Eignung von nominalisierten Satzkomplementen als Sprechaktberichte. Sofern Äußerungsverben überhaupt mit nominalisierten Satzkomplementen auftreten, ist zu beobachten, dass die NOML-Komplemente auf den Äußerungsgegenstand referieren und der implizierte Redebericht nicht weiter spezifiziert wird:

Sie erzählen von der Vertreibung durch die Rebellen und von ihren Mühen im Lager. (DWDS Zeit 2015)

\subsection{Potenzielle Konflikte bei der parallelen Lizenzierung von Satzkomplementen}

Aus obigen Ausführungen wird deutlich, dass es Konvergenzen bei den verschiedenen Komplementtypen geben kann, dass aber auch inkompatible Lizenzierungsbedingungen vorliegen. Die Konflikte bestehen einerseits zwischen den verschiedenen Satzkomplementtypen: So sind V2-Sätze und nominalisierte Satzkomplemente zumeist inkompatibel, insbesondere dort, wo es sich um Redeberichte handelt. Andererseits bestehen Konflikte auf Prädikatsseite. Intensionale Frageprädikate sollten keine Infinitivkomplemente selegieren. Faktive Verben sollten nicht mit V2-Sätzen und eingebetteten Polaritätsfragen auftreten. Äußerungsprädikate sollten Infinitiv- und nominalisierte Komplemente nur bedingt zulassen. Implikative Prädikate treten tendenziell nicht mit den finiten Komplementtypen auf. Mir geht es im Folgenden jedoch nicht um Selektionspräferenzen (siehe z.B. Rapp 2015 zu dass-Sätzen vs. Infinitiven, ebenso Brandt/Trawiński/Wöllstein 2016 und Rapp et al. 2017), sondern nur um eine grundsätzliche Kompatibilität.

Tabelle 6 zeigt einige sehr frequente Komplementationsmuster (belegt für jeweils mehr als 40 Prädikate) und die nicht belegten Komplementationsmuster in der ZAS-Datenbank.

Bei den Prädikaten, die nur mit zwei Komplementtypen belegt sind, wird die potenzielle Inkompatibilität von Infinitiv- und Interrogativkomplementen einerseits und V2-Sätzen und NOML-Komplementen andererseits bestätigt. Insgesamt gibt es jedoch keine absolut gültigen Implikationsbeziehungen zwischen den verschiedenen Komplementtypen. 
Tab. 6: Ausgewählte Komplementationsmuster laut ZAS-Datenbank

\begin{tabular}{lllllll}
\hline Beispiel & dass & V2 & INTER & INF & NOML & \#Prädikate \\
\hline betrauern & $\checkmark$ & & & & $\checkmark$ & 43 \\
& & $\checkmark$ & & & $\checkmark$ & 0 \\
abgewöhnen & $\checkmark$ & & $\checkmark$ & $\checkmark$ & & 0 \\
aufarbeiten & $\checkmark$ & & $\checkmark$ & & $\checkmark$ & 439 \\
abwägen & $\checkmark$ & $\checkmark$ & $\checkmark$ & $\checkmark$ & & 68 \\
abmahnen & $\checkmark$ & $\checkmark$ & $\checkmark$ & $\checkmark$ & $\checkmark$ & 257 \\
aufdecken & $\checkmark$ & $\checkmark$ & $\checkmark$ & & $\checkmark$ & 203 \\
anblaffen & $\checkmark$ & $\checkmark$ & $\checkmark$ & $\checkmark$ & & 65 \\
& & $\checkmark$ & $\checkmark$ & $\checkmark$ & $\checkmark$ & 43 \\
ahnen & $\checkmark$ & $\checkmark$ & $\checkmark$ & $\checkmark$ & $\checkmark$ & 435 \\
\hline
\end{tabular}

\subsection{Faktoren der syntaktischen Flexibilität}

Wie bereits angedeutet, kann die syntaktische Flexibilität durch Umdeutungen des Matrixprädikats oder semantische Anreicherung eines Infinitivkomplements begünstigt werden. ${ }^{12}$

Umdeutungen spielen auch bei Frageprädikaten, die eigentlich nicht mit Infinitivkomplementen und nur eingeschränkt mit dass-Sätzen auftreten sollte, eine Rolle. So können anfragen/nachfragen mittels Reinterpretation als direktives Prädikat mit der Bedeutung ,bitten‘ Infinitive einbetten:

(25) a. wenn die Feuerwehr bei uns angefragt hätte, ihre Anlage unterzubringen ... (DWDS BZ 2001)

b. Wir haben mehrfach nachgefragt, das Gold sehen zu dürfen. (ZDB 25230: DWDS PNN 2005)

Diese Reinterpretation ist nicht ungewöhnlich. Sprachvergleichend gibt es eine Reihe von Sprachen, in denen ,fragen' und ,bitten“ wie Englisch ask systematisch „kolexifiziert“ werden (siehe CLICS-Datenbank, List et al. 2014).

12 Auch bei V2-Sätzen (z.T. auch bei dass-Sätzen) finden sich modale Anreicherungen, z.B. deontische Modale bei direktiven Matrixprädikaten:

i. Und deshalb befahl er den Ammen und Pflegerinnen, sie sollten den Kindern Milch geben ... (ZDB 1574: DWDS K-Ge 1969). 
Ebenso kann man z.T. dass-Komplemente bei Frageprädikaten beobachten. In (26a) scheint eine Reinterpretation von erkundigen $\mathrm{zu}$,in Erfahrung bringen als Verschiebung der Handlungssequenz Fragen - Erfahren zugrundezuliegen.

(26) a. Allerdings hat er sich beim Badischen Handball-Verband inzwischen erkundigt, dass die Abmeldung einer Mannschaft 1200 Euro und den Abstieg in die Landesliga kostet. (ZDB 18718: IDS mm 2006)

b. Wer fragt danach, dass die barocken Fassaden in Berlin nur drei Fünftel des Baues umschlossen? (ZDB 24005: DWDS BZ 2004)

Anders gelagert ist ein Beispiel für (danach) fragen wie (26b), das bevorzugt in rhetorischen Fragen auftritt. Erwartungsgemäß referiert der dass-Satz nicht auf einen Fragesprechakt, sondern einen Sachverhalt, $\mathrm{zu}$ dem eine Frage gestellt werden kann. Hier liegt also ein anderer propositionaler Typ vor.

Der Bezug auf unterschiedliche Propositionen/Sachverhalte löst in vielen Fällen auch den Konflikt zwischen Komplementtypen, konkret zwischen V2-Sätzen und NOML-Komplementen. In (27a) bezeichnet das NOML-Komplement das von der Agitation intendierte Ziel, während der V2-Satz in (27b) den bei der Agitation geäußerten Sprechakt denotiert.

(27) a. Dadurch habe die Bevölkerung zum Sturz der Regierung aufgeputscht werden sollen. (ZDB 18343: DWDS K-Ze 1962)

b. Schließlich kommt er dahinter, daß es der Küchenmeister ist, der den Arbeitsinspektor aufputscht, er soll ihn nicht aus dem Kartoffelkeller rauslassen. (ZDB 1031: DWDS K-Be 1934)

\subsection{Koverte Lizenzierung}

In einigen Fällen scheint das Satzargument durch ein kovertes vermittelndes Prädikat lizenziert zu sein. In (28) ist anzunehmen, dass nicht beharren das Interrogativkomplement selegiert, sondern dass ein verstecktes Einstellungsverb wie wissen (also beharren zu wissen ...) als Lizenzierer des Komplements fungiert:

Ich möchte auch nicht darauf beharren, [wer nun wirklich für „Mad The Swine“ verantwortlich ist]. (ZDB 20569: IDS wpd 2011)

Ebenso ist abbringen, das Satzkomplemente, die eine zu erwartende Handlung charakterisieren, selegiert, kein plausibler Lizenzierer des V2-Satzes in (29). Hier 
könnte man alternativ ein verstecktes Einstellungsverb wie glauben oder ein Äußerungsprädikat wie behaupten als eigentliche Lizenzierer annehmen.

Nur war er nicht davon abzubringen, [er sei auf der anderen Seite des Berges heruntergefallen]. (ZDB 7: DNB 2006 S20 974566632)

Wie systematisch solche koverten Prädikate auftreten können, muss noch durch weitere Untersuchungen abgeklärt werden.

\section{Zusammenfassung und Ausblick}

Aus den vorausgegangenen Ausführungen dürfte deutlich geworden sein, dass die Polysemie eine wichtige Rolle in der Satzeinbettung spielt. Dies gilt sowohl für lexikalisierte Formen der Polysemie (Beispiel absehen) als auch für produktive Umdeutungsprozesse (wie der Instantiierung einer Sprechaktinterpretation bei bedauern). Weil das Einbettungsverhalten häufig nicht global dem satzeinbettenden Prädikat zugeordnet werden kann, sondern einer spezifischen Interpretation/ Variante zugeordnet werden muss, wird deutlich, dass der semantischen Selektion (S-Selektion) der Satzkomplemente eine zentrale Rolle zukommt.

Die Diskussion hat auch gezeigt, dass Faktoren wie Argumentstruktur/-realisierung des satzeinbettenden Prädikats, modale Operatoren im Matrixsatz, Komplementtyp (inkl. Komplementierer) und Verbmodus/Modalität, Tempus und evtl. weiteren Partikeln im eingebetteten Satz eng mit der Interpretation des satzeinbettenden Prädikats assoziiert sein können. Daraus ergibt sich unmittelbar die Frage, ob diese Faktoren die Lesart bestimmen oder umgekehrt die Lesart die Realisierung der genannten Eigenschaften lizenziert. Zur Beantwortung dieser Frage sind umfassende (auch sprachvergleichende) Untersuchungen zur Distribution und Systematik von Polysemiemustern bei satzeinbettenden Prädikaten erforderlich.

Die syntaktische Flexibilität der satzeinbettenden Prädikate wird durch verschiedene Faktoren begünstigt: (a) die Umdeutung des satzeinbettenden Prädikats, (b) die Realisierung von Satzargumenten in unterschiedlichen Argumentpositionen (wie bspw. bei Experiencer-Verben), (c) die semantische Anreicherung des Satzkomplements (insbesondere bei Infinitivkomplementen), (d) die Option, dem Satzargument z.T. unterschiedliche Propositionstypen zuordnen zu können, und (e) die Verwendung von koverten Lizenzierern. Die beiden ersten Faktoren sind Ausdruck der Polysemie eines satzeinbettenden Prädikats. 
Insgesamt zeigt sich, dass die Satzeinbettung aus einem komplexen Zusammenspiel von Modifikation bzw. Anreicherung des Matrixprädikats und Modifikation/Anreicherung des eingebetteten Satzes resultiert, was auch Konsequenzen für die theoretische Modellierung hat. Während Bogal-Allbritten (2016) für das generische Einstellungsprädikat nízin in Navajo noch überzeugend eine Analyse annehmen kann, in der das Einstellungsprädikat eher ein desemantisiertes Funktionsverb als Träger von Tempus etc. ist und die spezifische Interpretation durch Material aus dem eingebetteten Satz bestimmt wird, ist diese Analyse nicht ohne Weiteres auf Prädikate anderer Sprachen übertragbar, insbesondere dann nicht, wenn die Interpretation eng mit der Argumentstruktur/-realisierung des betreffenden Prädikats verknüpft ist. Nichtsdestotrotz müssen die Bedeutungskomponenten des Satzkomplements, die die Interpretation des Matrixprädikats beeinflussen, in die Modellierung aufgenommen werden.

Abschließend möchte ich anmerken, dass meine Ausführungen hier nur exemplarisch sind und die verschiedenen Lesarten polysemer satzeinbettender Prädikate noch systematischer abgeglichen werden müssen.

\section{Literatur}

Asher, Nicholas (2011): Lexical meaning in context. A web of words. Cambridge.

Askedal, John Ole (1997): drohen und versprechen als sog. ,Modalitätsverben“ in der deutschen Gegenwartssprache. In: Deutsch als Fremdsprache 34, S. 12-19.

Bogal-Allbritten, Elizabeth (2016): Building meaning in Navajo. Dissertation. Amherst.

Brandt, Patrick/Trawiński, Beata/Wöllstein, Angelika (2016): (Anti-)Control in German: Evidence from comparative, corpus- and psycholinguistic studies. In: Reich, Ingo/Speyer, Augustin (Hg.): Co- and subordination in German and other languages. In: Linguistische Berichte (LB). Sonderhefte 21, S. 77-98.

Cornillie, Bert (2004): The shift from lexical to subjective readings in Spanish prometer to promise' and amenazar ,to threaten'. A corpus-based account. In: Pragmatics 14, 1, S. 1-30.

Dipper, Stefanie (1997): Zur Selektion von Fragesatzkomplementen. (= Arbeitspapiere des Sonderforschungsbereichs (SFB) 340: Sprachtheoretische Grundlagen der Computerlinguistik 122). Stuttgart.

Engelberg, Stefan (2009): Blätter knistern über den Beton: Zwischenbericht aus einer korpuslinguistischen Studie zur Bewegungsinterpretation bei Geräuschverben. In: Winkler, Edeltraud (Hg.): Konstruktionelle Varianz bei Verben. (= OPAL-Sonderheft 4/2009). Mannheim, S. 75-97.

Heine, Bernd/Miyashita Hiroyuki (2008): Accounting for a functional category: German drohen ,to threaten'. In: Language Sciences 30, 1, S. 53-101.

Horn, Laurence (1978): Remarks on NEG-Raising. In: Cole, Peter (Hg.): Syntax and semantics. Bd. 9: Pragmatics. New York u.a., S. 129-220. 
Karttunen, Lauri (1977): Syntax and semantics of questions. In: Linguistics and Philosophy 1, 1, S. 3-44.

Kaufmann, Ingrid (1995): Konzeptuelle Grundlagen semantischer Dekompositionsstrukturen. Die Kombinatorik lokaler Verben und prädikativer Komplemente. (= Linguistische Arbeiten 335). Tübingen.

Levin, Beth/Rapoport, Tova R. (1988): Lexical subordination. In: Chicago Linguistic Society (CLS) 24, 1, S. 275-289.

List, Johann-Mattis et al. (2014): CLICS. Database of Cross-Linguistic Colexifications. Version 1.0. Marburg. Internet: http://CLICS.lingpy.org (Stand: 27.4.2017).

Nerlich, Brigitte et al. (Hg.) (2003): Polysemy. Flexible patterns of meaning in mind and language. Berlin.

Popp, Marie-Luise (2016): NEG-Raising in crosslinguistic perspective. Masterarbeit. Leipzig.

Pustejovsky, James (1995): The generative lexicon. Cambridge, MA.

Pustejovsky, James/Boguraev, Branimir (Hg.) (1996): Lexical semantics. The problem of polysemy. Oxford.

Rapp, Irene (2015): Zur Distribution von infiniten Komplementsätzen im Deutschen. Fragen, Fakten und Faktoren. In: Engelberg, Stefan et al. (Hg.): Argumentstruktur zwischen Valenz und Konstruktion. (= Studien zur Deutschen Sprache 68). Tübingen, S. 177-200.

Rapp, Irene et al. (2017): Lexikalisch-semantische Passung und argumentstrukturelle Trägheit - eine korpusbasierte Analyse zur Alternation zwischen dass-Sätzen und zu-Infinitiven in Objektfunktion. In: Deutsche Sprache 45, S. 193-221.

Reis, Marga (1997): Zum syntaktischen Status unselbständiger Verbzweit-Sätze. In: Dürscheid, Christa/Ramers, Karl-Heinz/Schwarz, Monika (Hg.): Sprache im Fokus. Festschrift für Heinz Vater zum 65. Geburtstag. Tübingen, S. 121-144.

Reis, Marga (2005): Zur Grammatik der sog. ,Halbmodale' drohen/versprechen + Infinitiv. In: D’Avis, Franz J. (Hg.): Deutsche Syntax. Empirie und Theorie. Symposium in Göteborg 13.-15. Mai 2004. (= Göteborger Germanistische Forschungen 46). Göteborg, S. 125-145.

Stiebels, Barbara (2010): Inhärente Kontrollprädikate im Deutschen. In: Linguistische Berichte 224, S. 391-440.

Stiebels, Barbara et al. (2017): ZAS database of clause-embedding predicates. Version 0.2. Mannheim. Internet: http://www.owid.de/plus/zasembed2017.

Talmy, Leonard (1985): Lexicalization patterns: Semantic structure in lexical forms. In: Shopen, Timothy (Hg.): Language typology and syntactic description. Bd. 3.: Grammatical categories and the lexicon. Cambridge, S. 57-149.

Traugott, Elizabeth C. (1993): The conflict promises/threatens to escalate into war. In: Berkeley Linguistics Society (BLS) 19, S. 348-358.

Troyke-Lekschas, Stephanie (2013): Korpuslinguistische Untersuchungen zum Phänomen der Satzeinbettung bei deutschen Geräuschverben. Magisterarbeit. Berlin.

Verhagen, Arie (2000): „The girl that promised to become something“: An exploration into diachronic subjectification in Dutch. In: Shannon, Thomas F./Snapper, Johan P. (Hg.): The Berkeley Conference on Dutch Linguistics 1997. The Dutch Language at the Millennium. (= Publications of the American Association for Netherlandic Studies 12). Lanham, S. 197-208.

Viberg, Åke (1983): The verbs of perception: A typological study. In: Linguistics. An Interdisciplinary Journal of the Language Sciences 21, 1, S. 123-162.

Wechsler, Stephen (2017): Clause embedding sound emission verbs. Vortrag auf der 39. DGfSJahrestagung, 8.-10.3.2017. Saarbrücken. 